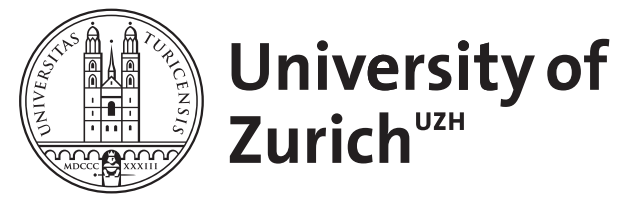

Zurich Open Repository and Archive

University of Zurich

University Library

Strickhofstrasse 39

CH-8057 Zurich

www.zora.uzh.ch

Year: 2010

Percutaneous implantation of an ASD occluder with intracardiac ultrasound

Enseleit, F ; Kretschmar, O ; Lüscher, T F

Posted at the Zurich Open Repository and Archive, University of Zurich ZORA URL: https://doi.org/10.5167/uzh-36411

Journal Article

Originally published at:

Enseleit, F; Kretschmar, O; Lüscher, T F (2010). Percutaneous implantation of an ASD occluder with intracardiac ultrasound. Cardiovascular Medicine, 13(9):290-291. 


\section{Percutaneous implantation of an ASD occluder with intracardiac ultrasound}

Frank Enseleit, Oliver Kretschmar, Thomas F. Lüscher

Cardiovascular Center Cardiology, University Hospital Zurich, Zurich, Switzerland

A 52-year-old female was admitted for further evaluation of a continuous heart murmur. Transthoracic echocardiography revealed a secundum type atrial septal defect (ASD II) with a size of $13 \times 16 \mathrm{~mm}$ and considerable left-to-right shunt $(\mathrm{Qp} / \mathrm{Qs}=2)$, as well as elevated pulmonary artery pressure (RV/RA pressure gradient $=38 \mathrm{~mm} \mathrm{Hg}$ ). Primary invasive evaluation and temporary closure of the ASD II using a sizing balloon showed a further elevation of the left atrial pressure/left ventricular end-diastolic pressure and consecutively of the pulmonary artery pressure, reflecting a left ventricular diastolic dysfunction. Therefore, the ASD II was left open and antihypertensive therapy with the angiotensin receptor blocker valsartan, at $80 \mathrm{mg}$ per day, was started. Three months later, the ASD II was successfully occluded using an Amplatzer ASD occluder, $26 \mathrm{~mm}$ in diameter, without any further elevation of the left ventricular filling pressures (fig. 1-4).

The atrial septum defect (ASD) is a common congenital lesion in adult patients. In $75 \%$ of cases, an ASD is a hole-like defect in the ostium secundum (ASD II) with a shunt flow from the "high pressure" left atrium to the "low pressure" right atrium [1]. However, an Eisenmenger reaction (right-to-left shunt) may, although rarely, occur in untreated patients after years of right heart overload with secondary pulmonary arterial hypertension and subsequent hypertrophy of the right atrium and ventricle. Patients usually develop symptoms (e.g. dyspnoea during exercise, palpitations, oedema, recurrent respiratory tract infections) in early adulthood. Other typical complications are atrial fibrillation, heart failure and embolic events [1].

For decades, surgical closure of ASD has been considered the treatment of choice. Since the mid 1990s transcatheter percutaneous device closure has established itself as the favoured approach [2]. Nowadays, 85-90\% of all ASDs in paediatric and adult patients can be closed percutaneously [3]. The main advantages of the percutaneous procedure are avoidance of open heart surgery and hence avoidance of anaesthesia (in case of intracardiac ultrasound as presented here, but not with the use of transoesophageal echocardiography) and of sternotomy with a thoracic of interest to disclose.
Figure 1

The device probe is introduced into the right atrium to visualise the defect size, location and adequacy of the septal rims (arrows). The device sheath is partially (dotted arrow) advanced into the left atrium. $\mathrm{RA}=$ right atrium; $\mathrm{LA}=$ left atrium; IAS = interatrial septum.

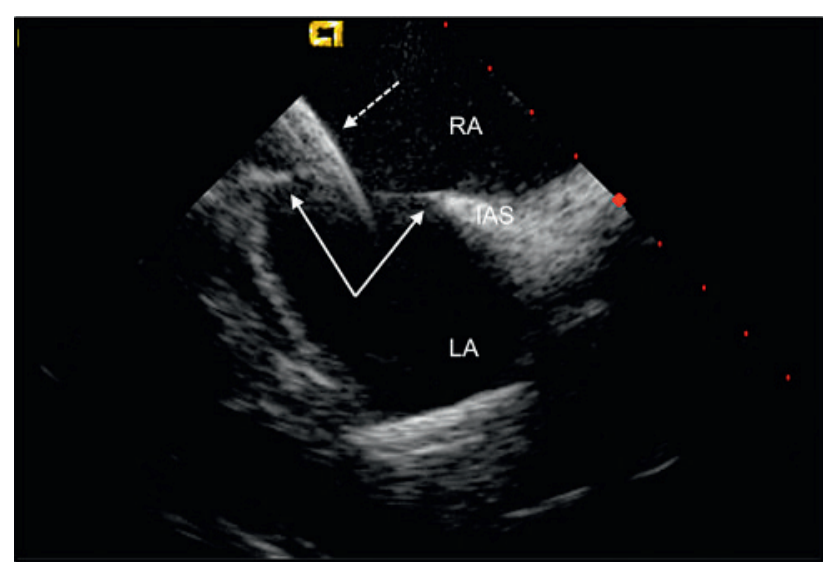

Figure 2

The intracardiac echocardiography shows the appropriately deployed Amplatzer septal occluder $26 \mathrm{~mm}$ (arrow) for atrial septal defect occlusion in short axis view.

$\mathrm{RA}=$ right atrium; $\mathrm{LA}=$ left atrium.



Correspondence:

Thomas F. Lüscher, MD, FRCP, FACC, FESC

Professor and Head of Cardiology

Cardiovascular Center Cardiology

University Hospital Zurich

Rämistrasse 100

$\mathrm{CH}-8091$ Zurich

Switzerland

cardiotfl@gmx.ch 
Figure 3

Intracardiac colour Doppler-echocardiography is used to rule out a periprosthetic leak after device deployment in short axis view. $\mathrm{RA}=$ right atrium; $\mathrm{LA}=$ left atrium .

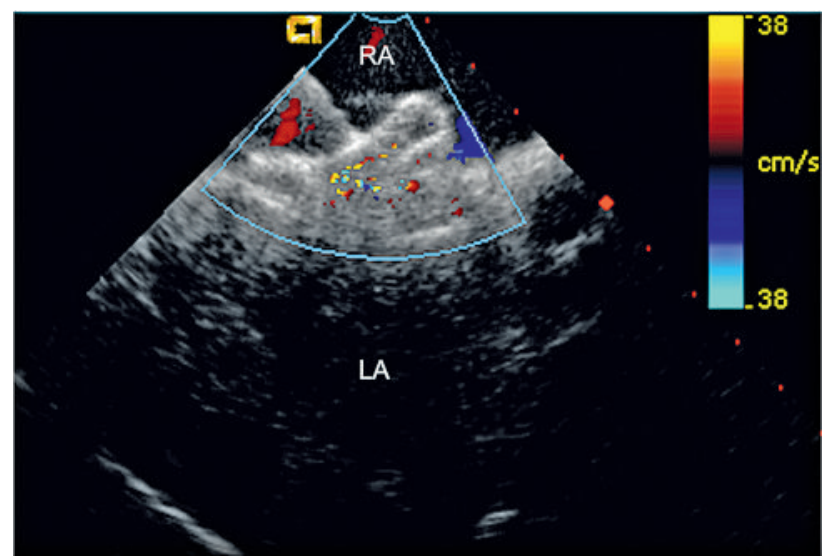

\section{Figure 4}

Injection of contrast medium during angiography confirms the correct position and configuration of the device (arrow) and excludes any right-to-left-shunt.

$\mathrm{RA}=$ right atrium; $\mathrm{LA}=$ left atrium.

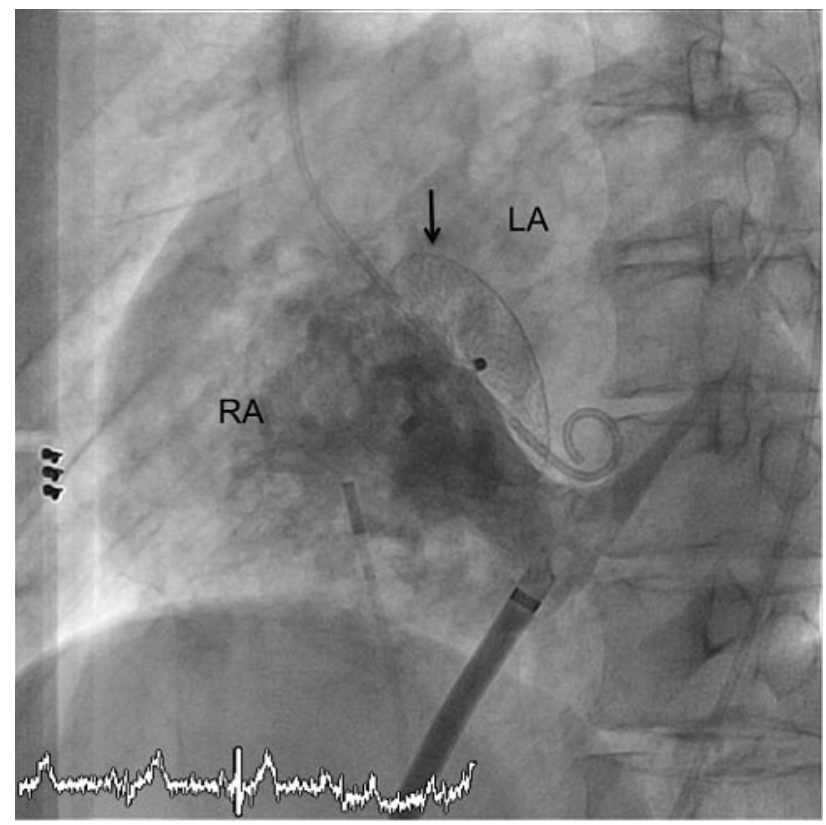

presented here with the help of intracardiac ultrasound even lends itself to an outpatient setting.

For the percutaneous procedure, large occlusion devices are used, because an ASD diameter of 30 to $40 \mathrm{~mm}$ is not uncommon. However, these devices may irritate the aortic root and the risk of malpositioning the device rises with increasing size. Both a patent foramen ovale and an ASD may be accompanied by a multiperforated septum or deficient interatrial rims, making device placement and anchoring more challenging [1]. An appropriate additional imaging modality (beside X-ray) during the procedure is mandatory to represent the exact anatomy of the defect and the device placement during the procedure. Nowadays, either transoesophageal or intracardiac echocardiography has been established for this purpose. Intracardiac echocardiography has several advantages such as improved patient comfort, avoidance of anaesthesia and hence easier logistics, as well as improved visualisation of the interatrial septum [4].

\section{References}

1 Hein R, et al. Atrial and ventricular septal defects can safely be closed by percutaneous intervention. J Interv Cardiol. 2005;18(6):515-22.

2 Ben Zekry S, et al. Percutaneous closure of atrial septal defect. JACC Cardiovasc Imaging. 2008;1(4):515-7.

3 Fischer G, et al. Experience with transcatheter closure of secundum atrial septal defects using the Amplatzer septal occluder: a single centre study in 236 consecutive patients. Heart. 2003;89(2):199-204.

4 Koenig P, Cao QL. Echocardiographic guidance of transcatheter closure of atrial septal defects: is intracardiac echocardiography better than transesophageal echocardiography? Pediatr Cardiol. 2005;26(2):135-9. 